\title{
Brief Note on System Signature and Long Term Method
}

\author{
Mehmet Gurcan ${ }^{1,}$ Ayse T. Bugatekin ${ }^{2 *}$ \\ ${ }^{1}$ Department of Statistics, University of Firat, Elazığ, Turkey,
}

\begin{abstract}
In this paper, the system signature of the system is creating by using the long-term method and then the transition matrices of each step are calculated. In this way, it is possible to have information about the reliability of the system after each unit becomes failure. Also, in this study, three systems are presented as examples.
\end{abstract}

\section{Introduction}

One of the materials used in the calculation of system reliability is the system signature, in recent years. There have been many studies on reliability of technical systems and system signature [1-7].System signature is obtained by calculating the probabilities of order statistics that disrupt the system from within the possible sequences, regardless of the distributions of the units that make up the system. The system signature is a probability vector as obtained from possible states. However, while the components of this probability vector are show the system corruption probability of order statistics up to last term, them last term is the form adapted to be 1 sum of probabilities. In fact, no matter what system, the system corruption probability of maximum order statistics is 1 always. In the study, if the $i$ th order statistic caused the disruption of the system, Bernoulli variable $\xi_{i}=0$ and otherwise $\xi_{i}=1$. Also, a step transition matrix $P(i)$, which represent a step transition between the corrupt and robust states $\{0,1\}$ of the system, has been established. Since the designed Markov chain is not homogeneous, the transition matrix at each step is different. So the system signature has been created for a given system and then the transition matrix for each step can be calculated. Thus, it is possible to have information about the reliability of the system after the failure of each unit.

\footnotetext{
Corresponding Author: ayseturan23@hotmail.com
} 
The long term method used in the study is designed with this principle. In this study, three systems are presented as examples.

\section{Construction of the Used Method}

The operating principle of a technical system is generally defined by some conditions placed on the units forming the system. In a series connected technical systems, if any of the units is corrupted the technical systems is corrupted. In parallel connected technical systems, if all units fail, the technical system is fail. Also, in $k$ out of $n$ technical system, if and only if at most $k$ machine are fail, the technical system is fail. In similar ways, these principles can be changed to define different technical systems and operating principles. It is evident from this that the order statistics of the distributions describing the working times of the units that constitute a technical system are very important in calculating the reliability of the technical system. If the system fails when the $r$ th unit fails in a technical system, the life-time of $r$ th order statistic describes the reliability of the system. Let $Y_{r}$ be $r$ th order statistic. The reliability of the system is calculated as follows,

$$
R=\operatorname{Pr}\left\{Y_{r}>t\right\}
$$

Accordingly, when the $r$ th disruption is occurs, the disruption of the system is expressed by $\xi_{r}=0$ and otherwise $\xi_{r}=1$. The transition probabilities between the states of the system are defined as follows,

$$
\alpha_{r}(i, j)=\operatorname{Pr}\left\{\xi_{r+1}=j \mid \xi_{r}=i\right\}, i, j=0,1
$$

In this case, the $P(r)=\left[\alpha_{r}(i, j)\right]$ matrix $\left\{\xi_{r}: r \geq 1\right\}$ that defined by the above probabilities, will be a step transition matrix of the non homogeneous stochastic process. However, let $1-\alpha_{r}=\operatorname{Pr}\left\{\xi_{r}=1\right\}$ be possibility of system operation when $r$ th disruption occurs and $\alpha_{r}$ possibility of system corruption when $r$ th disruption occurs. If a technical system consists of $\mathrm{n}$ units, then the n-component vector consisting of $\alpha_{r}$ components is denoted by $\eta=\left(\alpha_{r}: 1 \leq r \leq n\right)$. If $\alpha_{n}=1-\left(\alpha_{1}+\ldots+\alpha_{n-1}\right)$ is taken, the vector $\eta$ will be transformed into the system signature. At the same time, the following relationship exists between $\alpha_{r}$ possibilities,

$$
\left(\alpha_{r}, 1-\alpha_{r}\right) P(r)=\left(\alpha_{r+1}, 1-\alpha_{r+1}\right)
$$

It constitutes the idea that the basis of this designed analysis can be defined by a technical system $\left(\alpha_{r}\right)$ sequence. If the number of terms in this sequence is not limited by the 
number of units that make up the technical system, the sequence $\left(\alpha_{r}\right)$ is one that is convergent. This situation can only be applied to repairable systems. In literature reviews of repairable technical systems, it is assumed that the units have first performance after repair. However, this is unlikely in reality. In reality, even it can be repaired; each unit has a finite working cycle.

\section{Illustrative Examples}

1. The following table shows that the possible order of units of a technical system with a system signature $(6 / 24,14 / 2,4 / 24,0)$ and the order of disruption that causes the system to deteriorating system according to the specified order,

\section{Table 1.}

\section{Possible order/ (Order statistics that disrupt the system)}

\begin{tabular}{llllllllllllllllllll}
\hline 1 & 2 & 3 & 4 & $\mathbf{( 1 )}$ & 2 & 1 & 3 & 4 & $\mathbf{( 2 )}$ & 2 & 3 & 1 & 4 & $\mathbf{( 3 )}$ & 2 & 3 & 4 & 1 & $\mathbf{( 3 )}$ \\
1 & 3 & 2 & 4 & $\mathbf{( 1 )}$ & 3 & 1 & 2 & 4 & $\mathbf{( 2 )}$ & 3 & 2 & 1 & 4 & $\mathbf{( 3 )}$ & 3 & 2 & 4 & 1 & $\mathbf{( 3 )}$ \\
1 & 2 & 4 & 3 & $\mathbf{( 1 )}$ & 2 & 1 & 4 & 3 & $\mathbf{( 2 )}$ & 2 & 4 & 1 & 3 & $\mathbf{( 2 )}$ & 2 & 4 & 3 & 1 & $\mathbf{( 2 )}$ \\
1 & 3 & 4 & 2 & $\mathbf{( 1 )}$ & 3 & 1 & 4 & 2 & $\mathbf{( 2 )}$ & 3 & 4 & 1 & 2 & $\mathbf{( 2 )}$ & 3 & 4 & 2 & 1 & $\mathbf{( 2 )}$ \\
1 & 4 & 2 & 3 & $\mathbf{( 1 )}$ & 4 & 1 & 2 & 3 & $\mathbf{( 2 )}$ & 4 & 2 & 1 & 3 & $\mathbf{( 2 )}$ & 4 & 2 & 3 & 1 & $\mathbf{( 2 )}$ \\
1 & 4 & 3 & 2 & $\mathbf{( 1 )}$ & 4 & 1 & 3 & 2 & $\mathbf{( 2 )}$ & 4 & 3 & 1 & 2 & $\mathbf{( 2 )}$ & 4 & 3 & 2 & 1 & $\mathbf{( 2 )}$ \\
\hline
\end{tabular}

Thus, the $\eta$ vector $\eta=(6 / 24,14 / 2,1,0) \eta=(6 / 24,14 / 24,1,0)$ and the elements of the transition matrices, $\alpha_{1}(1,0)=4 / 9 \alpha_{2}(1,0)=1$.

2. In the second example, let's define the technical system $\alpha_{r}=1-\exp ((1-r) / l), l>0 r=1,2, \ldots$. In this case, each $\alpha_{r}(1,0)$ is obtained as $1-\exp (-1 / l)$. Here, $l$ is the adaptation constant of the long term method. 
3. In this example, let's examine a repairable technical system consisting of two units connected in parallel. Let the distribution of the operating times of both units be $F_{1}$ and $F_{2}$ and the distribution of the repair time be $G(u)$. After the system has started running, the first distorted unit will be repaired. When the second disruption takes place within the repair period, the system is corrupted. According to this, the operating time of the working unit for the deterioration of the system must be smaller than the repairing time of the units which are completed after the disruption in single row. In this case, the system signature consists of the following components,

$$
\alpha_{2 r-1}=0, \alpha_{2 r}=\operatorname{Pr}\left\{Y_{2 r}<U\right\}, r=1,2, \ldots
$$

Here, the components of the transition matrix are obtained as $\alpha_{2 r}(1,0)=\operatorname{Pr}\left\{Y_{2 r}<U\right\}$.

\section{Conclusion}

The operating principles of the technical systems described in the literature are increasing day by day according to the possibilities of technology and application areas. The identification of the technical system is equivalent to the identification of reliability in a way. In this study, the system signature is briefly discussed and a different method of using the constructed system signature has been put forward. Since the system signature is defined independently of the distribution and the operating time of the system, a timedependent dynamic analysis is not possible. However, this study can be done when the stochastic process that is introduced to describe the system in the study can be obtained with continuous parameters. In this regard, a technical system can be defined by rate matrix of a continuous parametric process. This definition will also lead to the introduction of many new systems.

\section{References}

1. P. K. Andersen, Q. Borgan, R. Grill and N. Keiding, Springer. New York (1992)

2. T. Avent, H. Haukaas, Reliab. Eng. and Syst. Safety, 58, 43-53 (1997)

3. R. Barlow, F. Proschan, Wiley. New York (1965)

4. A. Birolini, Springer. New York (1994)

5. B.V. Gnedenko, I. A. Ushakov, Wiley. New York (1995)

6. V.V. Kalashnikov, Syst. Anal. Model. Simul. 6, 833-851(1989)

7. J. Keilson, Springer. Berlin (2015) 